\title{
COVID-19 Infection in 8 Big Cities of India: The Dynamics of the Spread and Seropositivity
}

\author{
Zameer Shervani, Deepali Bhardwaj, Abdullah Sherwani, Intazam Khan and Umair Yaqub Qazi
}

\section{ABSTRACT}

The seroprevalence in the population of 8 big cities (Ahmedabad, Jaipur, Bangalore, Pune, Coimbatore, Surat, Visakhapatnam, and Nagpur) of India and the dynamics of the COVID-19 spread have been compared. The seropositivity data are of the self-referred residents only. The research is useful to know if the seroprevalence that occurred in the population has decreased the surge in infection in the second wave of the pandemic. The seroprevalence data are for the period July-December 2020 while the monthly new infections have been studied for July 2020-June 2021. For the cities: Visakhapatnam, Nagpur, Surat, Pune, and Coimbatore, the seropositivity of the population reached a plateau and then decreased. A decrease in seroprevalence did not result in higher infection rates. The seropositivity of Jaipur, Ahmedabad, and Bangalore showed a monotonous increase. A relationship between the plateau values of seroprevalence and infection rates could be established. More seroprevalence resulted in lower infection rates in cities (Bangalore, Visakhapatnam, Jaipur) and (Surat, Coimbatore, Pune) in the second wave of COVID-19 pandemic. The investigation of seroprevalence in the population will help in ramping up vaccination to eradicate the pandemic.

Keywords: COVID-19 India, second pandemic wave, seropositivity, seroprevalence, spread dynamics.

\section{INTRODUCTION}

As on July 1, 2021, large number of daily new cases $(>400,000)$ and deaths $(>8,000)$ were reported [1]-[3] around the globe, the pandemic has not slowed down and waned yet. One of the main reasons for fast spreading novel coronavirus is the delta variant. Slow vaccination, not wearing masks, and not physically distancing are the other reasons contributing to the caseload. As of on July 9, the count of $>186$ million total caseload and fatalities $>4$ million deaths were recorded. So, the pandemic is not under control. In Japan, novel coronavirus cases reached a peak of 6,505 new cases on May 15 in the fourth wave of infection, the cases started decreasing on June 24, a minimum of 1,438 cases were recorded. The cases started increasing again and on July 9, 1,753 cases were registered. The recent increase in the cases in Japan was attributed to the Delta variant of the virus. The Delta variant of coronavirus is also raising the case count [4] in the parts of the US where the number of vaccinated persons remained low. But in the US in general, because of vaccination drive, the number of new cases per day of 250,000 registered in January decreased to approximately 11,000 in the middle of June. But recently due to fast spreading the Delta variant, cases increased by $10 \%$ taking the daily case count to 12,500 . South
Submitted : July 21, 2021

Published : November 03, 2021

ISSN: 2593-8339

DOI: $10.24018 /$ ejmed.2021.3.6.985

\section{Z. Shervani*}

Food \& Energy Security Research \& Product Centre, Sendai, Japan.

(e-mail: shervani.nanotek@gmail.com) D. Bhardwaj

Centre for Skin and Hair Pvt. Ltd. D 305, Defence Colony, First Floor, New Delhi, India.

A. Sherwani

Interdisciplinary Unit of Nanotechnology, Zakir Husain College of Engineering and Technology, Aligarh Muslim University, Aligarh, India.

I. Khan

Flushing Hospital Medical Center, Flushing, New York, U.S.A.

U. Y. Qazi

Chemistry Department, College of Science, University of Hafr Al Batin, Hafr Al Batin, K.S.A.

*Corresponding Author
Korea's daily novel coronavirus caseload reached 800 , the highest in 6 months due to more contagious Delta strain of the virus [5]. The government has advised people to use masks even when indoor, discourage outings unless necessary, and ramped the vaccination drive. Elsewhere, South Africa in Africa with a 2.1 million caseload has 37\% of the continent's infections. Morocco and Tunisia have 9 and $8 \%$ of Africa's caseload, respectively. The higher rate of infection in South Africa is due to low vaccination, just 6\% population has been inoculated with one dose and two doses have been administered to only $2 \%$ of citizens making the country prone to the deadly virus.

On July 7, 2021, India crossed the COVID-19 cumulative case tally of $30,663,665$ ( $>30$ million). Only the US has higher cases than India with 33.5 million infections. The cumulative number of deaths in India was 404,211 $(>0.4$ million). Only the US and Brazil with 0.6 million and 0.5 million deaths, respectively were the first and second to the Indian fatalities. There were 459,920 active cases as on the above date constituting $1.5 \%$ of the total caseload. Though the positivity rate remained below $5 \%$ at 2.39 percent in receding the second wave of the pandemic in India. On the vaccine front, though in terms of the doses administered, India has inoculated the highest number of people but in terms of population ratio, only $17 \%$ of the people have 
received the first dose [6], [7]. Most (80\%) of the new COVID-19 cases were registered from 90 districts of Maharashtra (19), Tamil Nadu (15), Kerala (14), Odisha (11), Andhra Pradesh (10), Assam (9), and Karnataka (8). On the positivity side, 73 Indian districts are above $10 \%$ positivity while $5-10 \%$ positivity still exists in 65 districts which is the reason to worry. There are many violations in using masks and physical distancing. A survey showed that no use of mask in $24 \%$, limited use in $45 \%$, and proper use in $29 \%$ [8]. Until the pandemic is under control, observing COVID-19 appropriate behavior and strong vaccination drive are the only solution to keep the population healthy. Antibodies wane off in 7-12 months after the infection. The potential of vaccination for long term immunity and booster shots are being worked out by the researchers.

In the period February 11-May 9, the second wave of COVID-19 infections began in India in February, the pandemic was at the peak when the 7-day average of daily new infections increased 36 times [9]. After that daily new infections fell sharply till 45,000 cases then the decrease in new cases became slow. At present, the figure stands at 43,733 new cases in one day. Such a high number of infections in one day remained the reason to worry. Moreover, there is a fear that the daily new cases of more than 40,000 may become a baseline for the third wave. The baseline reported for the first wave was lower about 10,000 cases in one day. On the vaccine front, very recently Pfizer has suggested [10] booster shots based on the study that a third dose of vaccine was safe and raised the neutralizing antibodies titers by 5 -10-fold compared to the two-dose regime. Inoculating a booster shot regularly can solve the issue of waning out antibodies which is a major worry for herd immunity. This can open the countries and economies permanently functioning as were before the pandemic outbreak. The booster shot is also necessary in the context of the ultra-transmissible Delta variant and other future variants. Breakthrough cases were studied. In Britain, a study showed that the Pfizer vaccine was $88 \%$ effective in preventing the disease even from the Delta variant after the second shot. An Israel study however concluded that the Pfizer efficacy decreased to $64 \%$ against the Delta variant, though it was $93 \%$ good in keeping patients out of the hospital. From the management aspect, the idea of mixing and matching vaccines of different manufacturers has been proposed. However, World Health Organization suggested a cautious approach as the data are not available yet [11].

In Dharavi Mumbai (India) slums, COVID-19 infection generated antibodies were found in $45-57 \%$ of the residents. In another slum of Mumbai Cuffe Parade, the antibodies prevalence was $75 \%$. The $75 \%$ prevalence of the antibodies was enough to establish [12] herd or mass immunity in the slums that stopped the second wave of infection which happened in other parts of India. A large number of novel coronavirus cases registered in Kerala than in any other state of India was established because the state of Kerala has better health facilities thus residents have less immunity to the pathogen COVID-19 virus. The research results and data have been explained by "Health Index Theory" in two of the articles [13], [14]. COVID-19 virus viability and sanitization methods [15], risk of virus transmission from humans to pets [16] have been described in the references mentioned.
Researchers have published [17], [18] the data, at the onset of the pandemic, related to the COVID-19 detection, treatment, and vaccine development. Application of the world's fastest supercomputer "Fugaku" in picking the best suitable drugs and determining the famous " $3 \mathrm{Cs}$ " protocol has been given in an article published [19] earlier. In this research article, we studied the relationship between the seroprevalence and the caseload for the 8 big cities of India.

\section{MethodS}

Thyrocare Laboratories conducted SARS-CoV-2 IgG antibodies testing of self-referred residents of 8 Indian cities: Bangalore, Ahmedabad, Pune, Surat, Jaipur, Coimbatore, Visakhapatnam, and Nagpur. The survey was the initiative of the Canadian Institutes of Health Research and University of Toronto, Canada. A total of 448, 518 residents participated in the survey in the period June 2020 to December 2020. The COVID-19 infection rates in each city of India were taken from the website covid19india.org. The estimated current 2021 populations of different cities were used. More details of the survey conducted have been described in earlier articles [20], [21].

\section{RESUlTS AND DiscUSSION}

Fig. 1 is the seropositivity and monthly caseload (per million) of Bangalore city. Starting from July till December, seroprevalence showed a continuous increase. The seropositivity was $14.0 \%$, it increased to $42.3 \%$ in December. The new monthly caseload in the first wave of pandemic increased in July, August, and September. The caseload remained nearly the same in September and October. The cases decreased continuously from November 2020-February 2021. In the second wave of infection, a surge in the cases was noticed in March-May 2021 followed by a sharp decrease in June. In the first wave of infection, the monthly highest of nearly 8,000 per million cases were recorded in SeptemberOctober 2020. The infection rate decreased to a minimum of 531 cases per million in February 2021. The cases surged to a high of 32,000 per million in May 2021, then decreased to 3,962 cases in June.

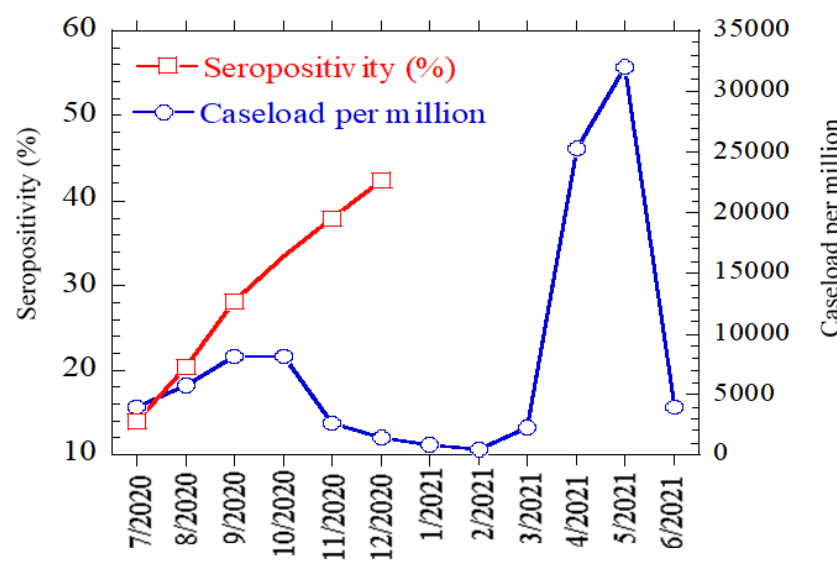

Fig. 1. Comparison of seropositivity and COVID-19 caseload in Bangalore.

Fig. 2 compares the seroprevalence and COVID-19 infection rate in Ahmedabad. The infection in Ahmedabad 
remained low. In July 2020, 683 new cases per million were recorded. Monthly new cases remained low till February 2021, in March cases started rising and reached a peak of 8,431 cases per million of population. In May and June, cases started decreasing again. In June, the lowest monthly cases of 284 per million were registered. The COVID-19 seropositivity in Ahmedabad residents increased linearly from a value of 11.1 to $48.4 \%$. The seroprevalence in $48.4 \%$ residents was not enough to stop the second wave of the pandemic as a large number of cases were registered in March 2021 and continued till May 2021.

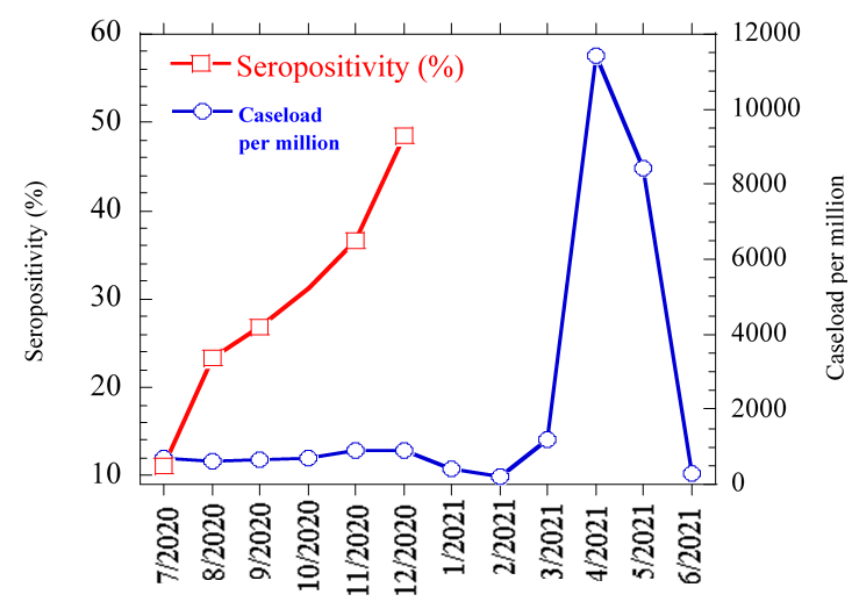

Fig. 2.Comparison of seropositivity and COVID-19 caseload in Ahmedabad.

Fig. 3 describes the seroprevalence and infection rate in the city of Jaipur. The seroprevalence increased sharply and monotonously from 13.0 to $63.8 \%$. A large population got infected in Jaipur compared to any other city in India. On the front of the new infection caseload, the monthly cases increased, in the first wave of infection, from July to November 2020 after that the cases decreased. Again, the second wave of infection cases surged in March 2021. In the first wave in November 2020, a maximum number of cases 3,600 per million were registered while in the second wave, a maximum of 18,442 cases were recorded in May 2021.

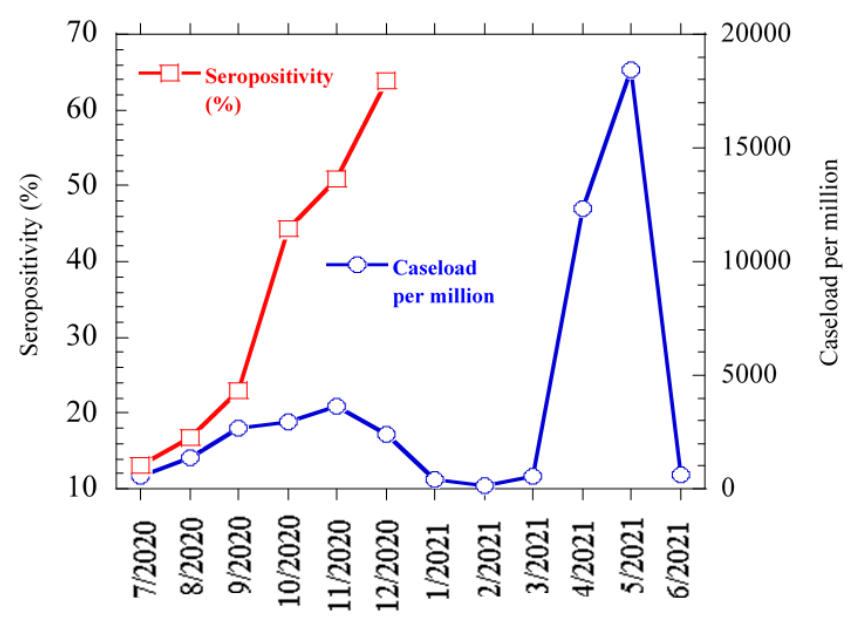

Fig. 3. Comparison of seropositivity and COVID-19 caseload in Jaipur.

The new monthly caseload in terms of cases per million and seroprevalence in Nagpur have been given in Fig. 4. The caseload increased from July 2020 to September 2020. After a peak in September cases started decreasing till January
2021. In February cases surge again and reached a peak in April then decreased till June 2021. In July cases were 1,161 per million, increased to 17,226 in September 2020. After the first wave was over, cases decreased to a minimum of 3,673 in January 2021. Cases surged to 5,471 in February reached a peak of 65,849 cases in April then decreased to 922 cases when the second wave ended in June. The seroprevalence nearly remained the same (nearly 10\%) in July and August. In September seropositivity increased to $32.1 \%$ after a plateau of $49.7 \%$ in October, the seroprevalence decreased to a value of $38.5 \%$ and then increased again in December to $41.8 \%$.

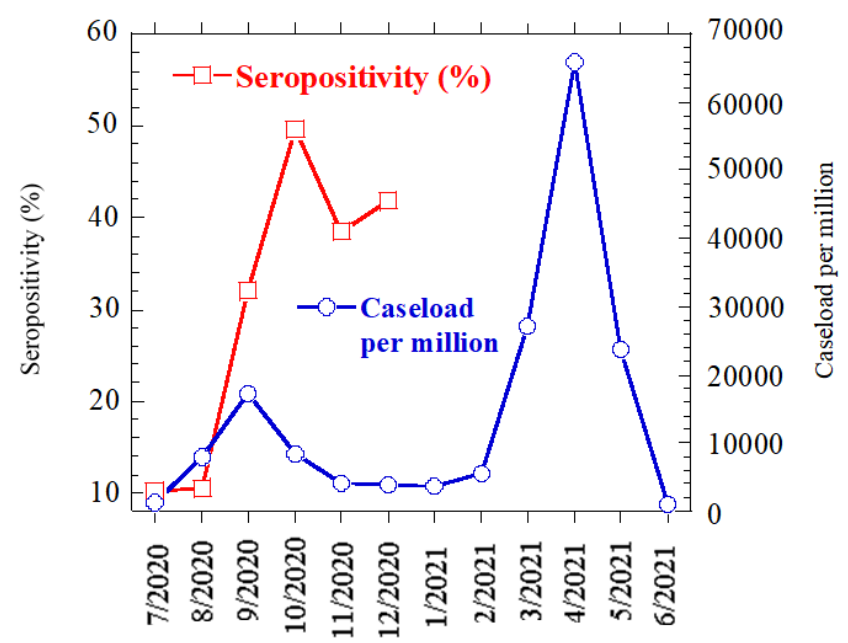

Fig. 4. Comparison of seropositivity and COVID-19 caseload in Nagpur.

The COVID-19 infection rate and seroprevalence in the city of Coimbatore have been shown in Fig. 5. In July and August 2020, seropositivity remained the same at $8.2 \%$. Seroprevalence increased linearly for the months September, October, and November. However, in December seropositivity decreased from 38.2 to $32.8 \%$. In the first wave of the pandemic, monthly infection rates increased for the months July, August, and September. When the first wave receded, cases decreased in the month October 2020 to February 2021. The cases started surging in March and reached a peak in June and then decreased in July when the second wave ended. The new cases in July were 1,529 per million. The cases increased to 5,920 in September, afterward started decreasing till February cases decreased to 489 and started surging to 32,888 cases in May 2021. After the second wave finished cases decreased to 17,502 in June.

As shown in Fig. 6, the number of monthly new cases showed a decrease in the city of Surat from July 2020 to February 2021. The second wave of pandemic is not clear and prominent in Surat as was the case in other cities of India. However, in the second wave cases surged in March 2021. After attaining a plateau in April 2021, cases decreased again in May and June. The cases per million in July 2020 were 1,098 . There was a negligible increase in September to 1,133 then decreased in October and the decrease continued till February 2021. In February the lowest number of cases of 181 were registered. In the second wave in April 6,647 cases were recorded in the peaked month. After the second wave was over in May the number of cases decreased to 3,446 and in June cases decreased further to 306 cases per million. The seroprevalence in the population of Surat was higher (32\%) in July compared to other cities. In August seropositivity 
increased to $47.9 \%$ after it decreased to $35.5 \%$ in September and further decreased in December to the lowest of $32.8 \%$.
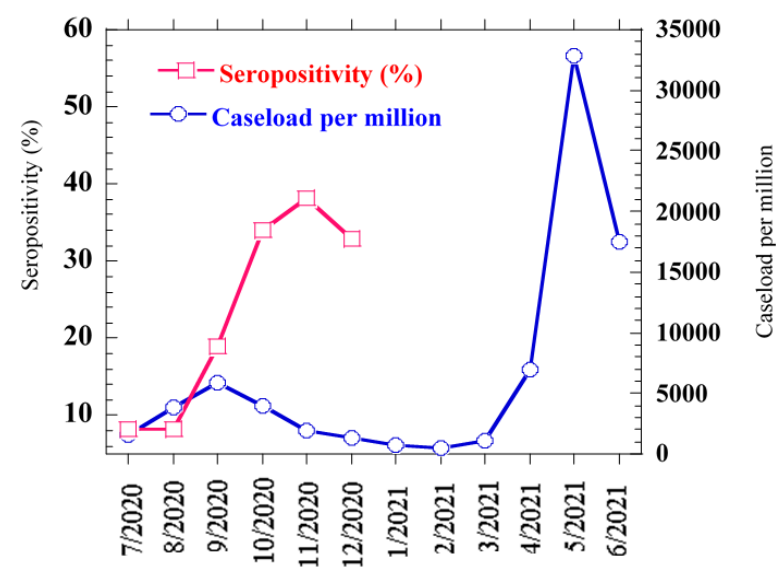

Fig. 5. Comparison of seropositivity and COVID-19 caseload in Coimbatore.

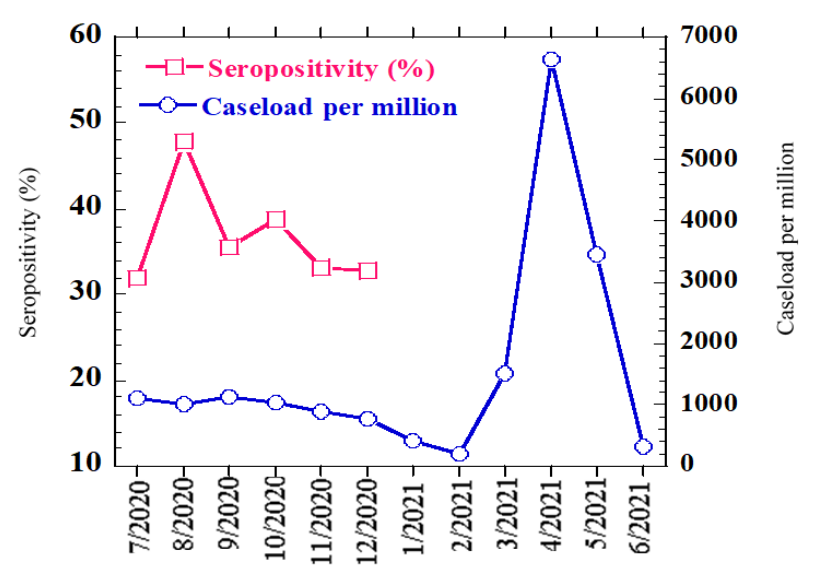

Fig. 6. Comparison of seropositivity and COVID-19 caseload in Surat.

The spread of novel coronavirus and seroprevalence in the Pune population has been plotted in Fig. 7. In the first wave of the pandemic, monthly new cases increased from July to September 2020. After September, cases started decreasing till January 2021. In February, cases increased, and the surge was noticed in March and cases peaked in April followed by a decrease in the number of cases in May and June. In July, the number of cases were 9,838 per million. Cases increased to a maximum of 17,346 in September in the first wave of the pandemic. When the first wave receded, cases started decreasing and dipped to the lowest of 2,332 in January 2021. In the second wave, cases surged to 18,594 in March and reached the highest of 45,147 in April, and in May and June cases decreased to 25,572 and 5,573, respectively. The seroprevalence showed a continuous increase from a value of $12.8 \%$ in July to $34.5 \%$ in October. In November the seroprevalence decreased to a value of $30.7 \%$ and showed a further decrease to a value of $24.4 \%$ in December.

Fig. 8 is the plot of the dynamics of the spread of the COVID-19 virus and seroprevalence in the population of Visakhapatnam. In Visakhapatnam, the first wave of the pandemic came earlier in August 2020 than in other cities of India which came in September. After the first wave was over, the monthly number of cases decreased until February 2021. Then in the second wave of the pandemic, the cases started surging again from March and the surge continued till the peak arrived in May. Again, the cases decreased when the second wave receded in June. The number of new cases per million increased from 4,646 in July to 11,785 in August. In February, cases became the lowest at 120 cases per million. The cases surged to a peak of 26,258 cases in May 2021 followed by a decrease in the cases to 5,204 cases in June. Antibodies prevalence in the Visakhapatnam population in July was $8.7 \%$, it increased to $51.5 \%$ in October then showed a decrease in November and December to 36.9 and $33.8 \%$, respectively.

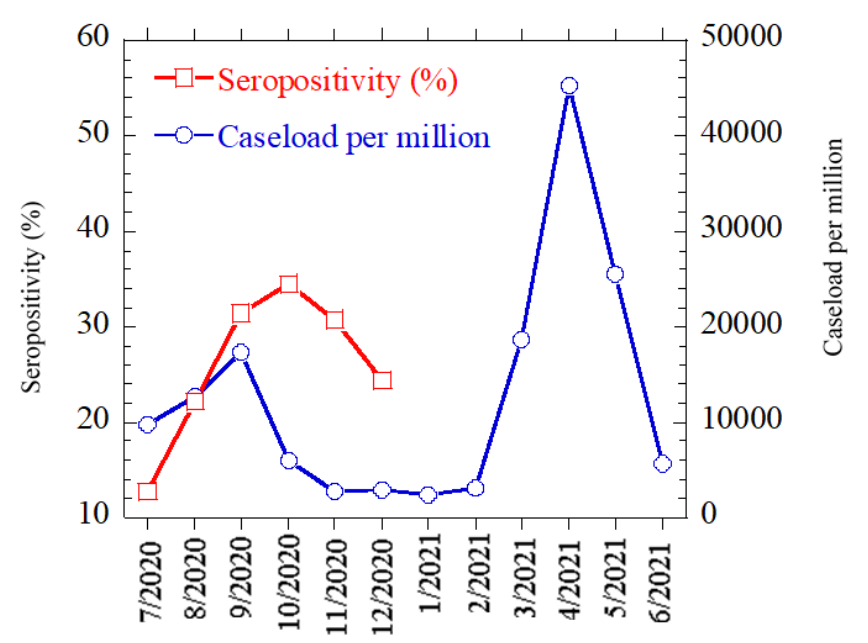

Fig. 7. Comparison of seropositivity and COVID-19 caseload in Pune.

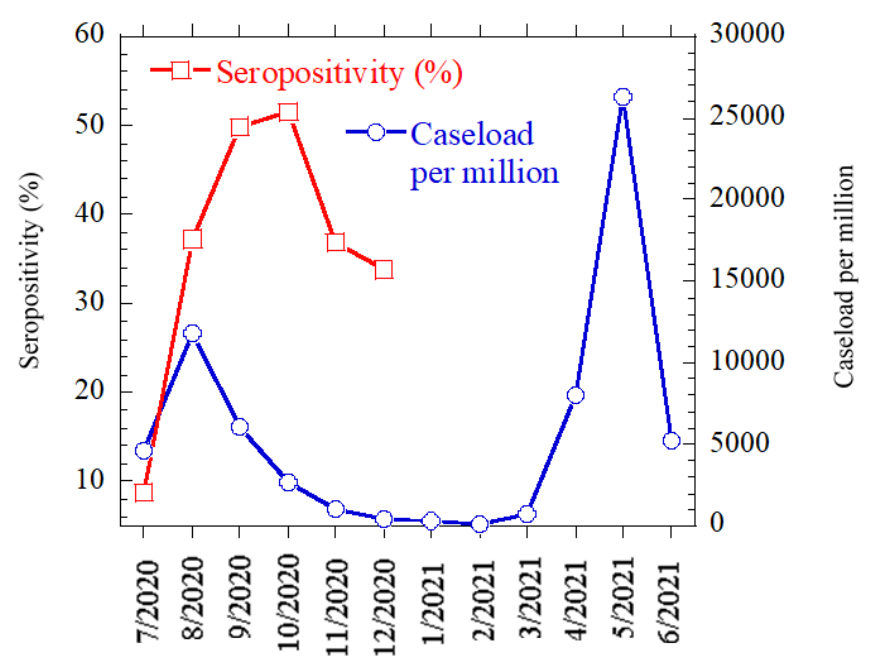

Fig. 8. Comparison of seropositivity and COVID-19 caseload in Visakhapatnam.

The effect of seroprevalence on the spread of the virus in four cities: Jaipur, Ahmedabad, Bangalore, and Visakhapatnam have been given in Fig. 9. The decreasing order of infections: Bangalore $(32,007)>$ Visakhapatnam $(26,258)>$ Jaipur $(18,442)$ were of the order of increasing the seropositivity at maximum: Bangalore (42.3\%) < Visakhapatnam $(51.5 \%)<$ Jaipur $(63.8 \%)$ which had justified that higher seroprevalence has suppressed the infections. The infection cases registered in Ahmedabad did not follow the above trend. The seroprevalence (48.4\%) in Ahmedabad has decreased the new infections to a larger extent of 11,413 cases than expected. Fig. 10 compares the effect of seropositivity on the daily new infection rate for the cities: Surat, Coimbatore, Nagpur, and Pune. The seroprevalence versus infection rate of different cities showed that the waning of the antibodies has not caused an increase in infection. The 
infection rate in the cities Surat, Coimbatore, and Pune followed the order of maximum seroprevalence existed and not that waned out. The order of seropositivity at the maximum value was Surat $(47.9 \%)>$ Coimbatore $(38.2 \%)>$ Pune $(34.5 \%)$ which might have decreased the maximum per million infections in a month in the order Surat $(6,647)<$ Coimbatore $(32,888)<$ Pune $(45,147)$ in the second wave of infection. Surprisingly, high seroprevalence (49.7\%) in Nagpur could not suppress the new infections as expected. The maximum daily new infections per million in a month in the second wave were still higher $(65,849)$ in Nagpur compared to the other cities. Several other factors apart from seroprevalence might have contributed to the large surge in the cases in Nagpur.

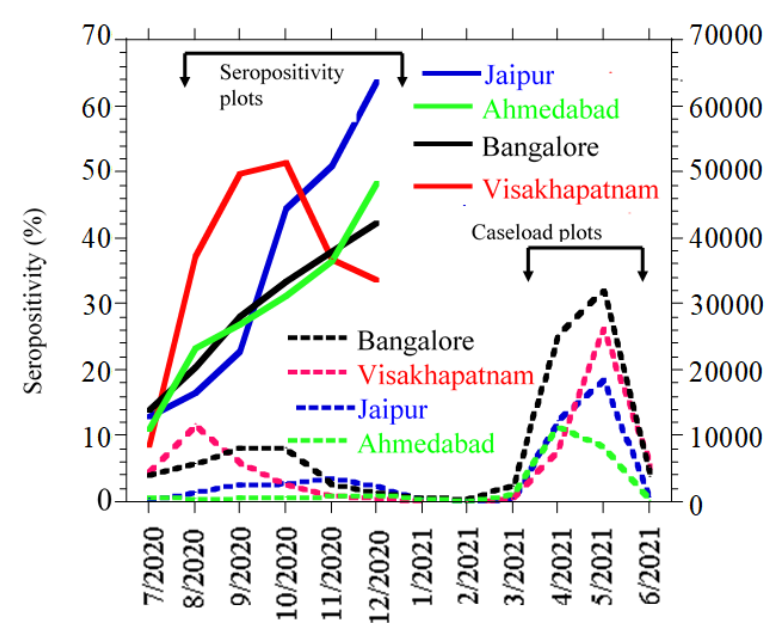

Fig. 9. Comparison of seropositivity and COVID-19 caseload in the cities Jaipur, Bangalore, Visakhapatnam, and Ahmedabad.

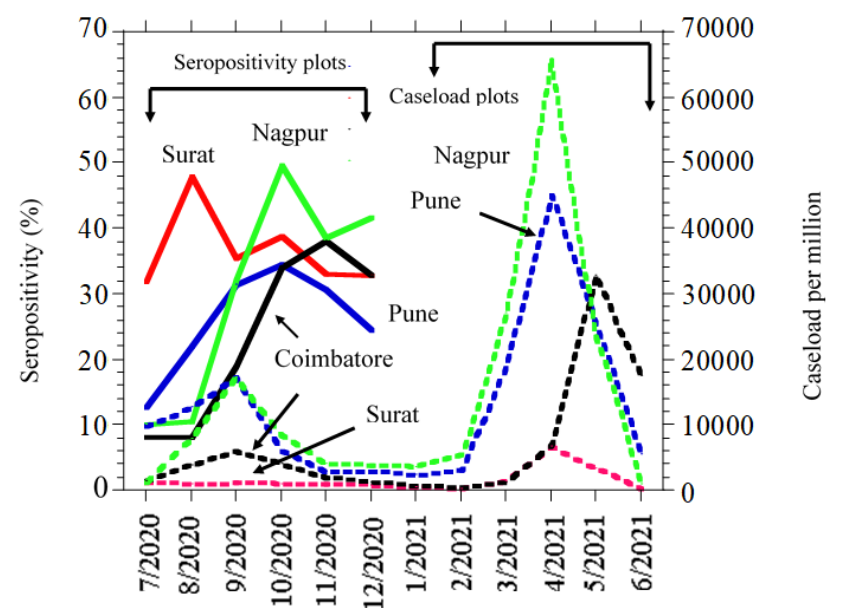

Fig. 10. Comparison of seropositivity and COVID-19 caseload in the cities Surat, Coimbatore, Nagpur, and Pune.

\section{STATEMENTS}

The data and results in this article are very reproducible. Author (Zameer Shervani, Ph.D.) is Director of Food \& Energy Security Research \& Product Center, Sendai, Japan. Authors have qualifications; Deepali Bhardwaj MBBS, MD, DVDL, M.Phil.; Abdullah Sherwani Bachelor of Technology (B.Tech.); Intazam Khan MBBS; Umair Yaqub Qazi Ph.D.

\section{ACKNOWLEDGMENT}

Corresponding author (Z.S.) is thankful to Professor Prabhat Jha for the access to the serosurvey data [21].

\section{REFERENCES}

[1] Coronavirus (COVID-19)-Google News (JHU CSSE COVID-19 DATA).

[2] Covid-19 not slowing down yet, Delta variant spreading rapidly:WHO's chief scientist Dr. Soumya Swaminathan, Hindustan Times, July 9, 2021.

[3] COVID-19 Cases in India and World: Country and City wise corona cases list, Times of India (indiatimes.com).

[4] White House prepares for COVID-19 outbreaks due to highly contagious Delta variants, Reuters, July 2, 2021.

[5] S. Korea's COVID-19 cases spike as Delta variant spreads, Reuters, July 2, 2021.

[6] 43,733 new Covid cases, 930 deaths registered in India in 24 hours; recovery rate rises to $97.18 \%$, The Times of India, July 7, 2021.

[7] India crosses the 3-crore mark in COVID-19 cases, lags behind in vaccinations, The Hindu, June 24, 2021.

[8] Number of fresh COVID-19 cases down but second wave not over yet: Ministry, The Hindu, July 6, 2021.

[9] Second Covid-19 wave lingering, or early signs of 3rd? Hindustan Times, July 9, 2021.

[10] Pfizer backs booster shots, US experts say no need yet, Hindustan Times, July 10, 2021.

[11] 'Caution folks': Top WHO scientist flags 'dangerous trend' of mixing Covid-19 vaccines, Times of India, July 12, 2021.

[12] Z. Shervani, D. Bhardwaj and R. Nikhat, "Dharavi Slums (Mumbai, India): The Petri Dish of COVID-19 Herd Immunity," European Journal of Medical and Health Sciences, 3(3): 38-41, 2021.

[13] Z. Shervani, "COVID-19 in Kerala: Health Index Theory," European Journal of Medical and Health Sciences, 3(2), 21-24, 2021.

[14] Z. Shervani, "COVID-19 in Kerala: The Dynamics of Spread and Health Index Theory," RAS Medical Science, 1(2), 1-3, 2021.

[15] Z. Shervani, I. Khan, N. Y. Siddiqui, T. Khan and U. Y. Qazi, "Viability of SARS-CoV-2 and Sanitization Methods," European Journal of Medical and Health Sciences, 3(1): 22-27, 2021.

[16] Z. Shervani, I. Khan, N. Y. Siddiqui, T. Khan and U. Y. Qazi, "Risk of SARS-CoV-2 Transmission from Humans to Pets and Vice Versa," European Journal of Medical and Health Sciences, 3(1): 34-38, 2021.

[17] Z. Shervani, I. Khan and U. Y. Qazi, "Sars-cov-2 delayed Tokyo 2020 olympics: Very recent advances in covid-19 detection, treatment, and vaccine development useful conducting the games in 2021," Advances in Infectious Diseases, 10(3): 56-66, 2020.

[18] Z. Shervani, I. Khan and U. Y. Qazi, "COVID-19 vaccine," Advances in Infectious Diseases, 10(3):195-210, 2020.

[19] Z. Shervani, I. Khan, T. Khan and U. Y. Qazi, "World's fastest supercomputer picks COVID-19 drug," Advances in Infectious Diseases, 10(3): 211-225, 2020.

[20] Z. Shervani, D.Bhardwaj and R. Nikhat, "COVID-19 Infection in India: Seropositivity versus the Dynamics of the Spread," European Journal of Medical and Health Sciences, 3(4):95-99, 2021.

[21] A. Velumani, C. Nikam, W. Suraweera, S. H. Fu, H. Gelband, P. E. Brown and P. Jha, "SARS-CoV-2 Seroprevalence in 12 Cities of India from July-December 2020," medRxiv 2021. 